Subscriber access provided by Brought to you by ST ANDREWS UNIVERSITY LIBRARY

\title{
Article
}

\section{Rotational Dynamics and Heating of Trapped Nanovaterite Particles}

Yoshihiko Arita, Joseph M. Richards, Michael Mazilu, Gabriel C. Spalding, Susan E. Skelton Spesyvtseva, Derek Craig, and Kishan Dholakia

ACS Nano, Just Accepted Manuscript • DOI: 10.1021/acsnano.6b07290 • Publication Date (Web): 01 Dec 2016

Downloaded from http://pubs.acs.org on December 6, 2016

\section{Just Accepted}

"Just Accepted" manuscripts have been peer-reviewed and accepted for publication. They are posted online prior to technical editing, formatting for publication and author proofing. The American Chemical Society provides "Just Accepted" as a free service to the research community to expedite the dissemination of scientific material as soon as possible after acceptance. "Just Accepted" manuscripts appear in full in PDF format accompanied by an HTML abstract. "Just Accepted" manuscripts have been fully peer reviewed, but should not be considered the official version of record. They are accessible to all readers and citable by the Digital Object Identifier (DOI $\left.{ }^{\circ}\right)$. "Just Accepted" is an optional service offered to authors. Therefore, the "Just Accepted" Web site may not include all articles that will be published in the journal. After a manuscript is technically edited and formatted, it will be removed from the "Just Accepted" Web site and published as an ASAP article. Note that technical editing may introduce minor changes to the manuscript text and/or graphics which could affect content, and all legal disclaimers and ethical guidelines that apply to the journal pertain. ACS cannot be held responsible for errors or consequences arising from the use of information contained in these "Just Accepted" manuscripts. 


\title{
Rotational Dynamics and Heating of Trapped
} Nanovaterite Particles

\author{
Yoshihiko Arita, ${ }^{\dagger, \ddagger}$ Joseph M. Richards, $₫$ Michael Mazilu, ${ }^{\dagger}$ Gabriel C. Spalding, $₫$ \\ Susan E. Skelton Spesyvtseva, ${ }^{\dagger}$ Derek Craig, ${ }^{\dagger}$ and Kishan Dholakia ${ }^{* \dagger, \dagger}$ \\ $\dagger S U P A$, School of Physics and Astronomy, University of St Andrews, North Haugh, Fife, \\ KY16 9SS, United Kingdom. \\ $\ddagger$ Molecular Chirality Research Centre, Graduate School of Advanced Integration Science, \\ Chiba University, 1-33 Yayoi, Inage, Chiba, 263-0022, Japan. \\ IIllinois Wesleyan University, Bloomington, Illinois 61701, USA. \\ E-mail: kd1@st-andrews.ac.uk \\ Phone: $+44(0) 1334463184$
}

\begin{abstract}
We synthesise, optically trap and rotate individual nanovaterite crystals with a mean particle radius of $423 \mathrm{~nm}$. Rotation rates of up to $4.9 \mathrm{kHz}$ in heavy water are recorded. Laser-induced heating due to residual absorption of the nanovaterite particle results in the superlinear behaviour of the rotation rate as a function of trap power. A finite element method based on the Navier-Stokes model for the system allows us to determine the residual optical absorption coefficient for a trapped nanovaterite particle. This is further confirmed by the theoretical model. Our data show that the translational Stokes drag force and rotational Stokes drag torque needs to be modified with appropriate correction factors to account for the power dissipated by the nanoparticle.
\end{abstract}




\section{Keywords}

vaterite; birefringence; spin angular momentum; optical torque; optical trapping; photothermal effect; hydrodynamics

Rotational control over optically trapped particles has gained significant prominence in recent years. The marriage between light fields possessing optical angular momentum and the material properties of microparticles has been useful to controllably spin microparticles in liquid, air and vacuum. ${ }^{1-7}$ The rotational degree of freedom adds new functionality to optical traps: in addition to allowing fundamental tests of optical angular momentum, the transfer of spin angular momentum in particular can allow measurements of local viscosity 2,8 and exert local stresses on cellular systems. ${ }^{9}$ Complementing bulk rheology, such techniques add key information about variation in material response, both with position and time-scale, providing information up to relatively high frequencies.

Spin angular momentum (SAM) of photons ( $\pm \hbar$ per photon) can be efficiently transferred to both dielectric birefringent microparticles and metallic nanoparticles. In the latter case, laser trapping of single gold nanoparticles induces dramatic heating that results in a temperature rise close to the critical temperature of water, $T_{c} \approx 650 \mathrm{~K}$, for a modest laser power of $50 \mathrm{~mW} .{ }^{5}$ Consequently a gas vapour around the nanoparticle reduces the local viscosity enabling the nanoparticle to rotate at frequencies of several $\mathrm{kHz} .{ }^{5}$ However such significant laser-induced heating may limit their wider use, both in biomedically relevant applications and for fundamental studies. ${ }^{10}$ To mitigate this heating issue, the fast rotation of gold nanocylinders was demonstrated with a rotation rate of $2 \mathrm{kHz}$ with a modest temperature rise of $22^{\circ} \mathrm{C}$ for a trapping laser power of $5 \mathrm{~mW}$, which was mediated through resonant light scattering. ${ }^{11}$ However, due to the large scattering cross-section, these nanoparticles were trapped only in 2D against a glass substrate, which limits the applicability of the technique. Furthermore, the use of any shape other than spherical would complicate the model 
to predict optical torques and the Stokes drag acting on the particle under consideration making it more challenging for use in microrheology.

In contrast to metallic nanoparticles or nanorods, a birefringent dielectric particle may induce a phase retardation between the ordinary and extraordinary components of the beam, resulting in a change in output polarisation and thus the SAM present in the circularly polarised (CP) incident light, leading to a torque upon, and rotation of, the particle. Notably, the key enabling dielectric material for many microrheological studies has been the development of micrometre-sized birefringent spheres. Previously, a range of birefringent microparticles have been used in optical traps including calcite fragments, ${ }^{1}$ fabricated quartz cylinders ${ }^{12}$ and liquid crystal probe microparticles. ${ }^{13}$ Solid vaterite microparticles, which possess a spherical morphology, have been particularly prominent in this regard, with sizes ranging from $1 \mu \mathrm{m}$ to $10 \mu \mathrm{m}$ in diameter. ${ }^{14}$ Notably microparticles with $4 \mu \mathrm{m}-5 \mu \mathrm{m}$ have dominated the reported experiments to date. Rotation rates for vaterite microparticles have ranged from a few tens of $\mathrm{Hz}$ up to a few hundred hertz in liquid ${ }^{8}$ and $\mathrm{MHz}$ rates in vacuum. ${ }^{4}$ Importantly, a number of key opportunities for studies, e.g. in levitated optomechanics and in micro-rheological properties of cells, would be opened up with the synthesis of submicrometre-to-nanometre scaled vaterite spheres allowing both higher rotation rates and higher trap oscillation frequencies. This property may be useful for future studies in levitated optomechanics. ${ }^{15,16}$ For biological studies, reducing the size of vaterite crystals would facilitate more facile uptake by cells by endocytosis or pinocytosis and may aid cell viability. ${ }^{17}$

Furthermore, little information is available regarding the heating effects of these types of rotating nanoparticles in optical traps. While isothermal Brownian motion is well understood, non-equilibrium rotational Brownian motion of heated nanoparticles has only been discussed theoretically. ${ }^{18}$ Importantly, this study shows that heating affects the translational and rotational degrees of freedom and frictional coefficients of a hot nanoparticle in different ways. Our results agree with this conclusion. Previously, Parkin et al. considered a 
shell-model for calculating the rotation rate of the fluid shells as a function of distance from the centre of the heated microparticle, which depends on the temperature gradient along the distance. For micrometre-sized vaterite crystals, a temperature increase of $66^{\circ} \mathrm{CW}^{-1}$ was inferred for trapping at the near infra-red wavelength of $1064 \mathrm{~nm} .{ }^{9}$ However this model simulates the dynamics of fluid around the microparticle rather than the microparticle itself. Therefore there is scope for a detailed understanding of the role of particle absorption and its influence on the ultimate nanoparticle dynamics for both the rotational and translational degrees of freedom.

In this article, we have tailored our experiments to study the absorption of the rotating particle itself. This is in contrast to previous studies where both absorption in the liquid and particle both needed to be taken into consideration. We present an optical trapping system with a $532 \mathrm{~nm}$ laser beam, and using a medium (heavy water) that has virtually no absorption at this laser wavelength. In turn this means we can truly isolate the temperature distribution around the particle, due to particle absorption, from any issue arising with regard to laser absorption by the surrounding medium. Such a system is invoked to test a limit to spin angular momentum transfer to nanoparticles and to clearly reveal the connection between heating and the rotational and translational degrees of freedom.

We demonstrate the synthesis, optical trapping and rotation of birefringent nanovaterite crystals in heavy water. We record rotation rates up to $4.9 \mathrm{kHz}$ in liquid. We study the rotational and translational dynamics of nanovaterite and measured the trap rotation frequency and trap corner frequency as a function of laser power. In turn they are both indicative of the nanoparticle surface temperature and corresponding viscosity. Our numerical model for the experimental data shows that the translational Stokes drag force is higher than that of the rotational Stokes torque. We define correction factors for both degrees of freedom to account for the power dissipated by the nanoparticle. Our study represents a detailed understanding of the heating effects on rotating dielectric nanoparticles held in an optical trap. Such information is crucial not only for micro/nano-rheological studies of biological 
media, but also for future studies in levitated optomechanics in vacuum.

\section{Results and Discussion}

Vaterite is a polymorph of calcium carbonate $\left(\mathrm{CaCO}_{3}\right)$ with a spherical morphology and is polycrystalline, which is typically composed of $20-30 \mathrm{~nm}$ sized nanocrystals. However, such nanocrystals are highly oriented in such a way that the direction of the optical axis is in a hyperbolic distribution throughout the formed vaterite crystals. ${ }^{19}$ The effective refractive index as a whole particle is anisotropic and therefore vaterite is considered to be effectively a positive uniaxial birefringent material. Previously, the main drive for synthesis of nano-scale vaterite has been for use as localised delivery systems. We synthesize nanovaterite crystals via the co-precipitation method in $83 \%$ ethylene glycol solvent. The fabrication protocol for nanovaterite crystals is described elsewhere. ${ }^{20}$ It is to be noted for such small vaterite crystals, the procedure varies significantly from that for standard vaterite microparticles ${ }^{14}$ (see Methods section for details). Fig. 1 shows the images of nanovaterite crystals acquired by a scanning electron microscope (SEM) at different scales. The mean particle radius was found to be $423 \mathrm{~nm} \pm 63 \mathrm{~nm}(\sigma)$ with a surface roughness of $13 \mathrm{~nm}\left(\sigma_{r}\right)$ for a given batch of nanoparticles (see Supplementary Fig. S1 for a histogram of the distribution counts of particle radius). In experiment these particles are further filtered to reduce the polydispersity to achieve an effective standard deviation $\sigma_{e} \leq \pm 25 \mathrm{~nm}$.

We note that we may isolate particles much smaller in size using appropriate filtration to eliminate larger ones. The current method for the synthesis of nanovaterite can yield a large quantity of submicron crystals with polydispersity, which allows us to preselect the particle size desired. Fig. S1 shows the particle size distribution of nanovaterite crystals, which covers particle radii smaller than $250 \mathrm{~nm}$. The efficiency of obtaining such smaller particles is low but we obtain an acceptable rate of around $5-10 \%$ of the total quantity.

In general calcium carbonate minerals such as calcite or vaterite are chemically unstable 


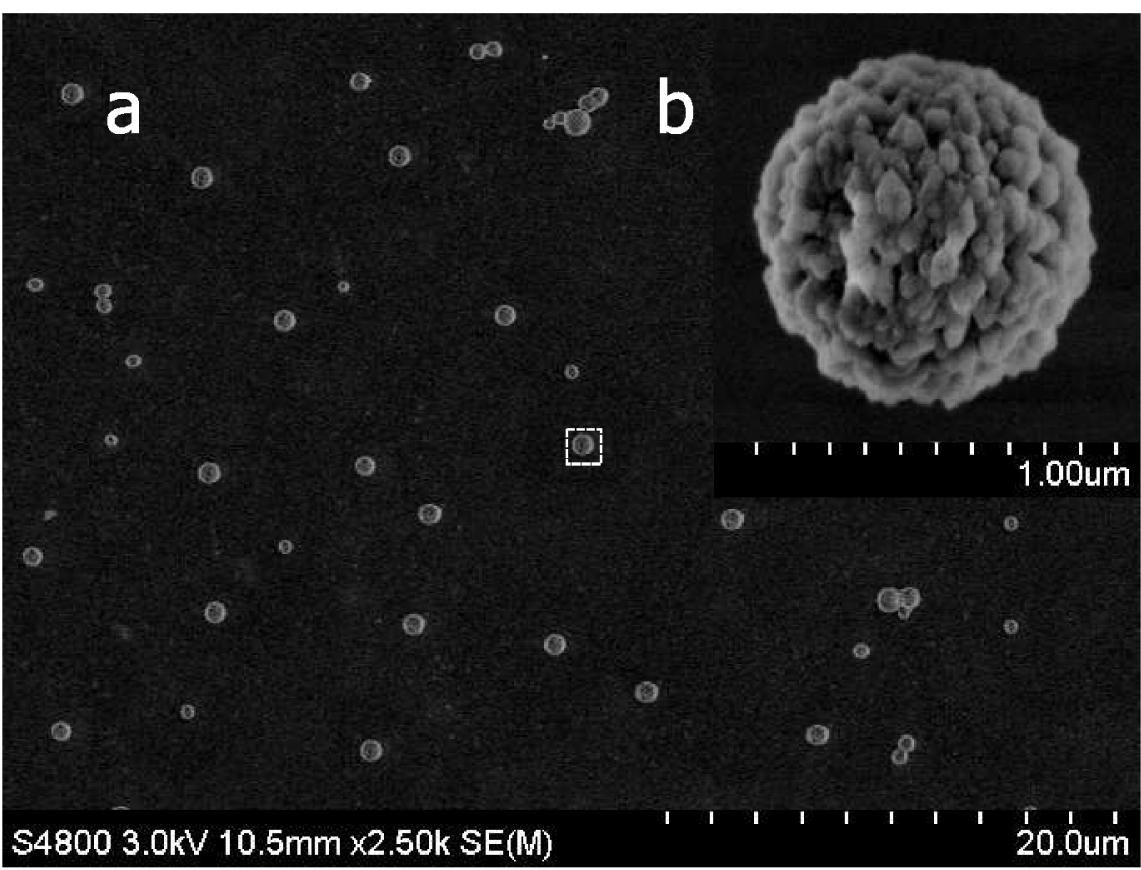

Figure 1: SEM images of nanovaterite crystals. (a) A selection of nanoparticles (b) An expanded view of the enclosed field with dashed line boundary.

and can be dissolved in water. These nanovaterite particles can typically last around two hours for experiments. Beyond this time frame, vaterite may change its optomechanical behaviour or simply may not rotate at all. This time frame, however, is sufficient for meaningful measurements in most applications in microrheological studies. In addition, we have not observed any temperature effect on the degradation of the particle over the range of optical powers tested.

When CP light passes through the birefringent vaterite particle, the polarisation change results in a change in the angular momentum of the trapping laser beam, related to the relative phase shifts along the ordinary and extraordinary optical axes, and the effective thickness of the material. Since the photon polarisation state $\sigma$ changes during traversal of a birefringent particle, the particle must experience a torque: $\tau_{\text {opt }}=(\hbar \Delta \sigma)(P / \hbar \omega)=$ $\Delta \sigma_{\mathrm{CP}} P / \omega$, where $\hbar$ is the reduced Planck's constant, $\Delta \sigma_{\mathrm{CP}}=\left(P_{L}-P_{R}\right) / P$ the change in circular polarisation of the beam with $P_{L}$ and $P_{R}$ the left and the right circularly polarised components of the beam power, $P$ the total optical power at particle, and $\omega$ the angular 
frequency of light. Thus, the particle will accelerate and reach a terminal angular velocity when the optical torque is balanced by the hydrodynamic rotational drag, which, for an isolated sphere, is given by the rotational Stokes drag torque, $\tau_{\text {drag }}=8 \pi \mu r^{3} \Omega$, where $\mu$ is the viscosity of the surrounding medium, $r$ the particle radius, and $\Omega=2 \pi f_{\text {rot }}$ the angular frequency of the rotating vaterite particle. By equating $\tau_{\text {opt }}=\tau_{\text {drag }}$, the angular frequency of the particle is given by $\Omega=\Delta \sigma_{\mathrm{CP}} P / 8 \pi \mu r^{3} \omega \propto P$ at the steady-state temperature. Thus the rotation rate, $f_{\text {rot }}$ of the particle depends linearly on the optical power, $P$, if there is no heating process in the system. Fig. 2 shows the power spectrum of a nanovaterite particle trapped by a CP beam with an optical power of $3188 \mathrm{~mW}$. (see Methods section for details and Supplementary Fig. S2 for optical set-up.) The rotation frequency $f_{\text {rot }}$ of $4.9 \mathrm{kHz}$ is recorded together with the high harmonics of this frequency.

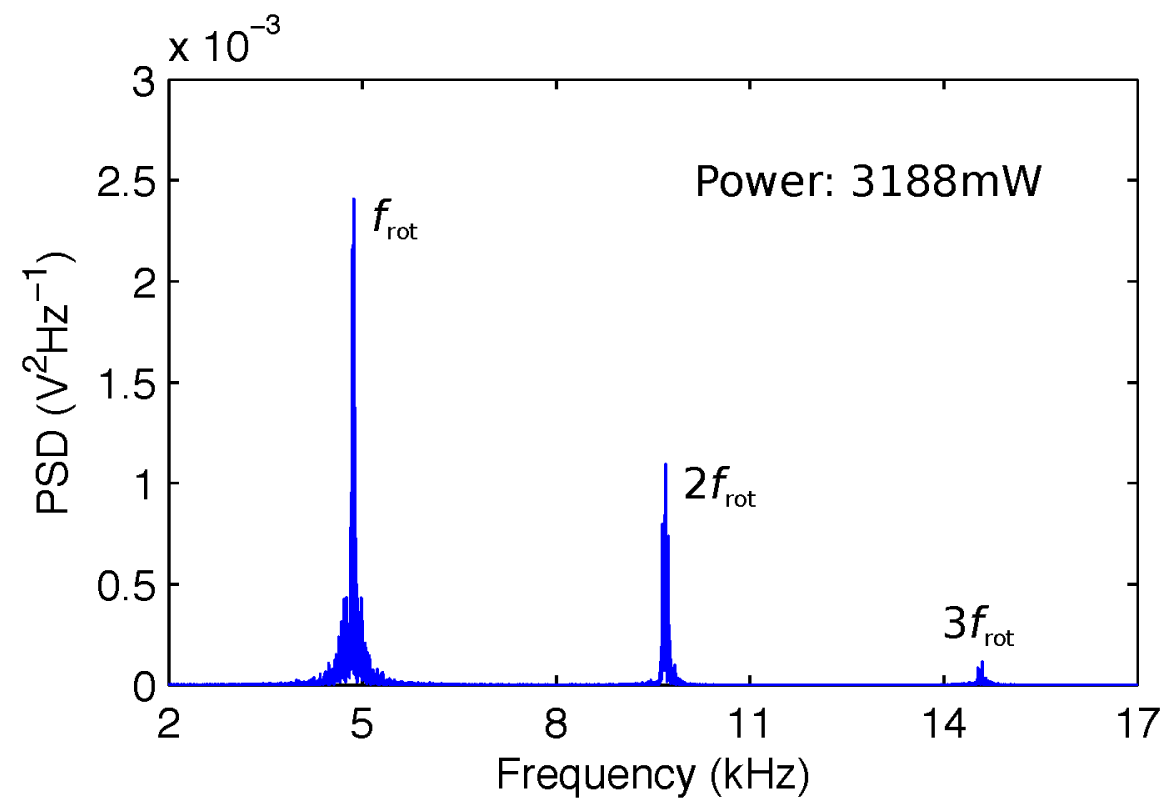

Figure 2: Power spectral density (PSD) of a rotating nanovaterite particle showing a rotation rate, $f_{\text {rot }}$ of $4.9 \mathrm{kHz}$ and its high harmonics of $2 f_{\text {rot }}$ and $3 f_{\text {rot }}$.

In contrast to CP light, when a birefringent uniaxial crystal, such as vaterite is trapped in a linearly polarised (LP) beam, the optical axis of the crystal aligns with the electric field, which is perpendicular to the propagation direction of the beam resulting in no particle rotation. In this case the particle refracts the light and the refracted photons impart 
momentum to the particle. The vaterite particle experiences a restoring force towards the focus of the beam, $F_{\text {grad }}=\kappa x$, where $\kappa$ is the trap stiffness and $x$ the distance of the particle travelled from the trap. Over a long time-scale the particle is confined, however, over shorter time-scales the particle is free to diffuse. This corresponds to fluidic damping of the particle's motion in the free-diffusion case, but the confining potential acts as a cut-off to the particle displacement. A sphere of radius $r$, translating in a fluid of viscosity $\mu$, experiences a drag force $F_{\text {drag }}=6 \pi \mu r v$, where $v$ is the velocity of the particle. In the over-damped case, $F_{\text {drag }}$ is balanced by the optical restoring force, $F_{\text {grad }}$, yielding a time scale for traversal of the trap $\tau=x / v=6 \pi \mu r / \kappa$. The roll-off frequency from the power spectral density (PSD) is then given by $f_{\mathrm{c}}=\tau^{-1}=\kappa / 6 \pi \mu r \propto P$. Thus the corner frequency, $f_{\mathrm{c}}$ depends linearly on the optical power, $P$, assuming the steady-state temperature of the system.

Fig. 3a shows the rotation rate, $f_{\text {rot }}$ (blue open circles) and the corner frequency, $f_{\mathrm{c}}$ (green open squares) as a function of optical power using the same nanovaterite particle (423 $\mathrm{nm}$ in radius) trapped by CP (for rotation) and subsequently LP (for translation) light fields. Superlinear behaviour is observed both in $f_{\text {rot }}$ and in $f_{\mathrm{c}}$ depending on the optical power, which suggests the local heating of the surrounding medium caused by the residual optical absorption of the nanovaterite crystal. We have modelled the rotation rate and the corner frequency of the nanovaterite particle taking into account the fluid dynamics drag torque/force acting on the nanoparticle, which is dependent on the nanoparticle motion, either rotational or translational. The drag torque and force are related to the viscosity of the surrounding water which is itself temperature dependent. ${ }^{21}$ Considering the residual absorption of the nanovaterite particle, we can determine, using heat transfer equations, ${ }^{22}$ the water temperature gradient and therefore its local viscosity. A laminar Navier-Stokes model $^{23}$ is used which can then deliver the overall drag torque or force for the rotational and translational motion of the nanoparticle.

We have used finite element method (implemented in COMSOL ${ }^{\circledR}$ ) to calculate the overall drag torque or drag force for different residual optical absorption powers. ${ }^{24}$ Assuming the 

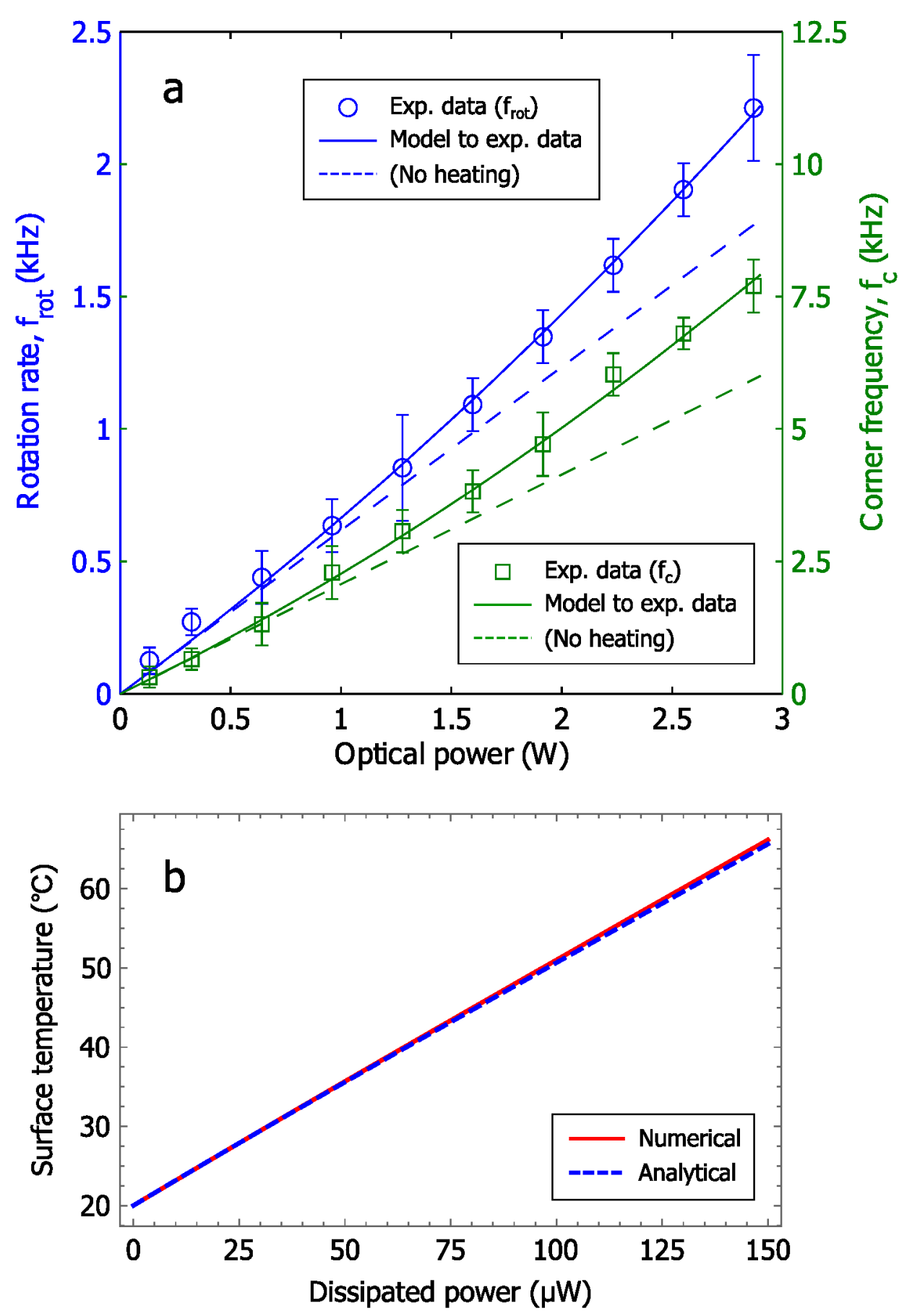

Figure 3: Nanoparticle rotation rate, $f_{\text {rot }}$ and trapping corner frequency $f_{\mathrm{c}}$ as a function of the incident optical power. (a) Measured data ( $f_{\text {rot }}$ represented by blue open circles and $f_{\mathrm{c}}$ by green open squares) together with model results ${ }^{24}$ (curved solid lines take heating into account and dashed lines neglect heating). (b) Nanoparticle surface temperature, $T_{s}$ as a function of the dissipated power using a finite element approach ${ }^{24}$ and an analytical approximation (Eq. 1). 
proportionality of optical torque (blue dashed line in Fig. 3a) and trap stiffness (green dashed line in Fig. 3a) with the incident power it is possible to fit the numerical model with the experimental data and determine the residual optical absorption coefficient, which links the incident power to the absorption of the nanovaterite particle. Further, using the theoretical model it is possible to determine the surface temperature, $T_{s}$ of the sphere depending on the absorbed/dissipated power (Fig. 3b). In a first order approximation, the surface temperature of the nanoparticle can be calculated using

$$
T_{s}=\frac{Q_{\mathrm{abs}}}{4 \pi r k\left(T_{s}\right)}
$$

where $Q_{\mathrm{abs}}$ is the absorbed/dissipated power and $k\left(T_{s}\right)$ the thermal conductivity of heavy water. ${ }^{25}$ Solving this equation for the surface temperature, $T_{s}$ gives a analytical approximation which is in a good agreement with the full numerical model using COMSOL ${ }^{\circledR}$ (Fig. 3b). The simultaneous fit to both the rotation rate (blue solid line in Fig. 3a) and the corner frequency (green solid line in Fig. 3a) data implies a global absorption coefficient of $1.59 \times 10^{-5}$ for nanovaterite with a particle radius of $423 \mathrm{~nm}$, i.e. for an incident beam with a power of $1 \mathrm{~W}$, the nanoparticle dissipates $13.4 \mu \mathrm{W}$, which leads to the surface temperature of $24.2^{\circ} \mathrm{C}$ equivalent to a rise of $4.2^{\circ} \mathrm{CW}^{-1}$. To show the validity of this surface temperature for different particle sizes, we study the lower and upper limit cases of $r_{\mathrm{L}}=373 \mathrm{~nm}$ and $r_{\mathrm{U}}=473 \mathrm{~nm}$ based on the particle size distribution of $r=423 \mathrm{~nm} \pm 50 \mathrm{~nm}\left(2 \sigma_{e}\right)$. We found that $T_{s}=24.2^{\circ} \mathrm{C}$ for an incident power of $1 \mathrm{~W}$ remains unchanged within this particle size range. This is due to the fact that the change in particle size affects both the power absorbed/dissipated by the particle and its surface area in such a way that maintains $T_{s}$ within these limits. We note that nanovaterite particles used in experiment are not spheres (see Fig. 1b). Here we assume that the error due to the non-spherical form of the nanovaterite particles is taken into account in the polydispersity error $\left(\sigma_{e}\right)$. Indeed, the drag coefficient of a rotating non-spherical nanoparticle can, in most circumstances, be approximated by a spherical nanoparticle with a different effective radius. 
It is worth contrasting the transfer of angular momentum from light by absorption with that by phase retardation (polarisation change). For a particle that absorbs power $P_{\text {abs }}$ from an incident circularly polarised beam, the resultant torque is $\tau_{\text {abs }}=P_{\text {abs }}\left|\sigma_{z}\right| / \omega$, where $\sigma_{z}= \pm 1$ for circularly polarised light and $\omega$ the angular frequency of the trapping light. By using a global absorption coefficient of $1.59 \times 10^{-5}$ for nanovaterite (particle radius of $423 \mathrm{~nm}$ ), we obtain $P_{\text {abs }}=0.5 \times 10^{-20} \mathrm{Nm}$ for an incident power of $1 \mathrm{~W}$. The rotational Stokes drag torque $\tau_{\text {drag }}=8 \pi \mu r^{3} \Omega$ can be equated to $\tau_{\text {abs }}$ to obtain $\Omega$, which results in a rotation rate of $2 \mathrm{~Hz}$ for this incident power. Considering the total observed torque and the resultant rotation rates, we conclude the torque by absorption is negligible, which suggests that the internal birefringence of nanovaterite crystals is responsible for the rotation of these particles.

We also note that nanovaterite particles can be rotated at a rate about $10 \mathrm{~Hz}$ with a low laser trapping power of $10 \mathrm{~mW}$, which would cause a negligible rise in particle temperature of about $0.04^{\circ} \mathrm{C}$. For cellular studies, nanometric sized particles such as nanovaterite are attractive as they can be more preferably internalised by cells via endocytosis or pinocytosis $^{17,26}$ potentially without compromising cell viability. Whilst we do concentrate on studies at $532 \mathrm{~nm}$, we remark that we are readily able to rotate these particles at $780 \mathrm{~nm}$ and $1064 \mathrm{~nm}$, leading to their future use for biomedical applications. Controlled rotation of nanovaterite (even at a low rate of a few tens of $\mathrm{Hz}$ ) without heating would allow quantitative measurements of local viscosity of intracellular environments ${ }^{9}$ and exerting local stresses on cellular systems. $^{27}$

The finite element model gives also access to the local temperature and viscosity variation around the nanoparticle. ${ }^{24}$ Fig. 4 a shows the variation of the temperature (blue solid line) and the corresponding viscosity (red solid line) depending on the distance from the nanoparticle surface. The temperature of a fluid varies with the distance from the nanoparticle surface, which means that there will be a non-uniform distribution of fluid viscosity around the sphere. This non-uniform distribution implies the need for a correction factor when calculating the 
Stokes drag coefficient experienced by the nanoparticle compared to the homogeneous case. Further, depending upon whether it is the rotational or translational degree of freedom under consideration, we need different correction factors.
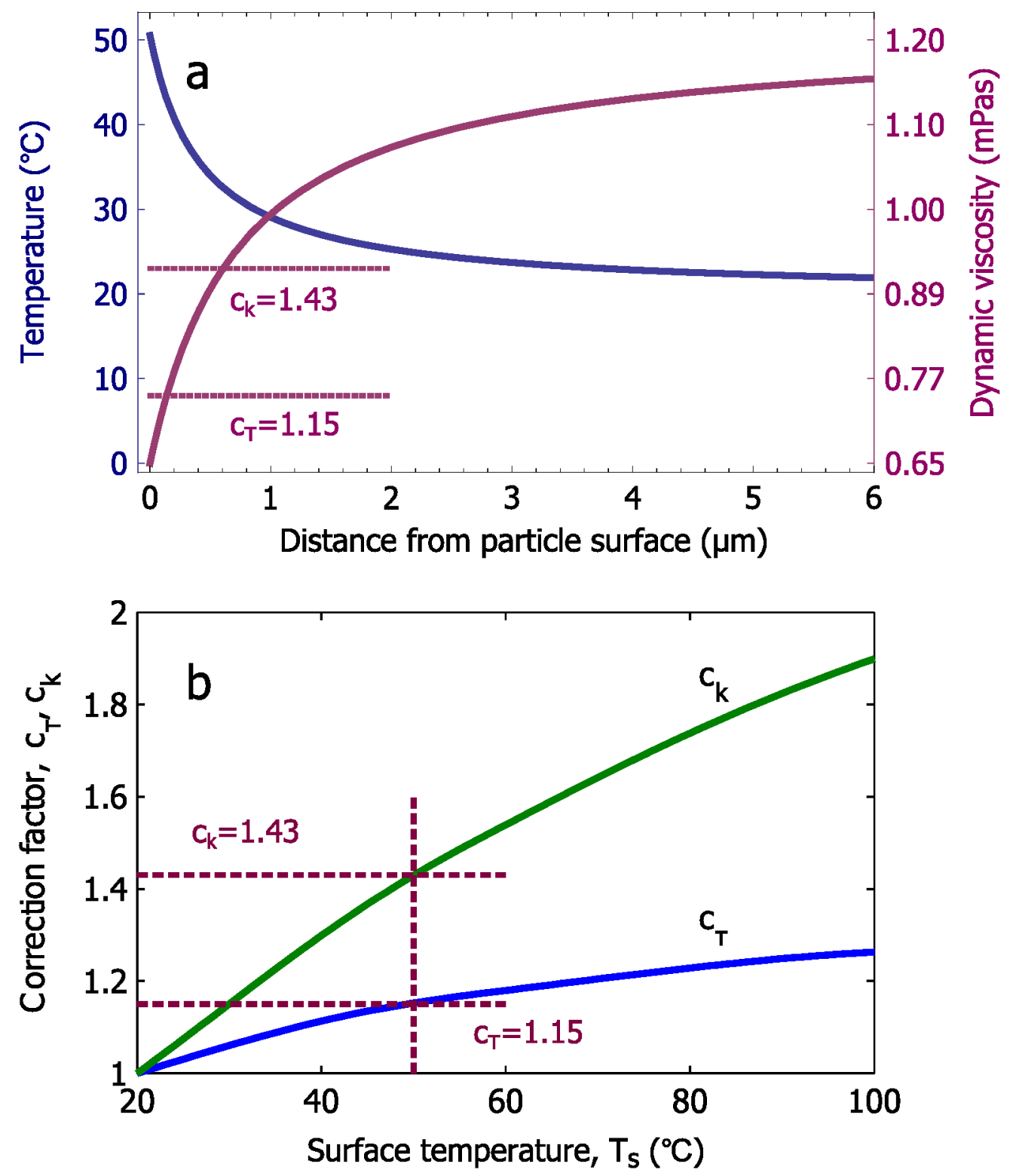

Figure 4: Temperature dependent dynamic viscosity correction factor. ${ }^{24}$ (a) Radial temperature profile and the corresponding dynamic viscosity of heavy water surrounding a nanoparticle dissipating $100 \mu \mathrm{W}$. (b) Viscosity correction factors (as defined in the main text) for rotational (blue solid line) and translational (green solid line) motion of the nanoparticle (radius of $423 \mathrm{~nm}$ ) as a function of its surface temperature.

To take into account the effect of the non-uniform viscosity profile around a trapped 
nanoparticle, we introduce a correction factor, $c_{\tau}\left(T_{s}\right)$ and $c_{k}\left(T_{s}\right)$ into the Stokes drag formula for both the rotational and translational motion.

$$
\begin{aligned}
\tau_{\text {drag }} & =c_{\tau}\left(T_{s}\right) 8 \pi \mu\left(T_{s}\right) r^{3} \Omega \\
F_{\text {drag }} & =c_{k}\left(T_{s}\right) 6 \pi \mu\left(T_{s}\right) r v
\end{aligned}
$$

where $\Omega$ and $v$ define the rotation rate and linear motion of the nanoparticle. Fig. $4 \mathrm{~b}$ show the behaviour of these correction factors as a function of the surface temperature considering our particular experimental setup. When the surface temperature of the nanoparticle, $T_{s}$ is maintained at the same temperature as the surrounding water $\left(20^{\circ} \mathrm{C}\right)$, i.e. a uniform temperature distribution around the nanoparticle, these correction factors are unity. In this case, $c_{\tau}=c_{k}=1$. As $T_{s}$ increases (creating a non-uniform distribution of temperature thus fluid viscosity), the translational Stokes drag force will have a higher correction factor than that of the rotational motion, i.e. $c_{k}>c_{\tau}>1$. An interesting case occurs when $\mathrm{Yb}^{3+}$-doped crystals are trapped by a tunable, near-infrared continuous-wave laser. ${ }^{28}$ In this case, heat can be dissipated through the crystal lattice across the solid-liquid interface by anti-Stokes (blue-shifted) photons following upconversion of $\mathrm{Yb}^{3+}$ electronic excited states mediated by the absorption of optical phonons. In such a case, $T_{s}$ can be colder than the surrounding medium, where the correction factors satisfy $c_{k}<c_{\tau}<1$.

It should be noted that these correction factors are dependent on the particle radius and the surface temperature of the particle. Further, the difference between the rotational and translational Stokes drag correction factors may be understood by considering the nanoparticle's temperature in its immediate environment. When rotating the nanoparticle is only in contact with liquid at the surface temperature whereas during translation it explores a temperature variability. 


\section{Conclusion}

In summary, we have synthesised nanovaterite crystals with a mean particle radius of $423 \mathrm{~nm}$. We have demonstrated trapping and rotation of such nanoparticles, achieving rotation rates up to $4.9 \mathrm{kHz}$. Superlinear behaviour of the rotation rate as a function of laser power is observed. This behaviour arises from the variation of the local viscosity as a consequence of local heating of the medium due to the absorption of the birefringent nanovaterite crystal. We have developed a full numerical and theoretical model to determine the surface temperature of the nanoparticle by simultaneously fitting the model to the experimental data based on the trap rotation frequency and the trap corner frequency. Our data reveals that the nanoparticle experiences a different Stokes drag torque or force depending on whether we consider rotational or translational motion, which is in a good agreement with the theoretical prediction of the rotational hot Brownian motion. The data allow us to determine the correction factors for the local viscosity for both the rotational and translational motion of the nanoparticle. The use of nanovaterite particles opens up new studies for levitated optomechanics in vacuum as well as microrheological properties of cells or biological media. For these latter studies, nanovaterite offers prospects of microviscosity measurements in ultra small volumes and, due to its size, potentially simpler uptake by cellular media. ${ }^{17}$

\section{Methods}

Nanovaterite synthesis. Our highly spherical nanovaterite particles are grown via the controlled precipitation of concentrated solutions of calcium chloride $\left(\mathrm{CaCl}_{2}\right)$ and sodium carbonate $\left(\mathrm{Na}_{2} \mathrm{CO}_{3}\right) \cdot{ }^{20}$ The final diameter of these nanovaterite spheres can be controlled by altering a number of variables. This includes the concentration of the reactants, the reaction time and the speed of rotation during mixing. A key issue in the fabrication of these spheres is avoidance of rapid recrystallisation into the calcite phase, which can occur due to the enhanced solubility of the particles as the particle size decreases. To avoid such 
problems, ethylene glycol was added to the water used as the solvent for this reaction. This reduces the crystal growth rate and diminishes the chance of calcite phase nucleation. For the nanoparticles used in this study, 0.33 $\mathrm{M}$ solutions of calcium chloride and sodium carbonate were prepared in $83 \%$ ethylene glycol $(\mathrm{v} / \mathrm{v})$, mixed at room temperature while stirring at $500 \mathrm{rpm}$ for 3 hours. The resultant precipitate was obtained through centrifugation at $3000 \mathrm{rpm}$ for 20 minutes prior to washing with ethanol and repeating twice. The resultant nanoparticles were then dried for at least 1 hour at $60^{\circ} \mathrm{C}$. In a typical batch of nanovaterite samples, the mean particle radius was found to be $423 \mathrm{~nm} \pm 63 \mathrm{~nm}(\sigma)$. See Fig. 1 for SEM images.

Experimental setup. A schematic diagram is shown in Supplementary Fig. S2. The experimental apparatus comprises of a trapping laser beam (Lighthouse Photonics Inc., SproutG: continuous wave, wavelength $532 \mathrm{~nm}$, power up to $10 \mathrm{~W}$ ) propagating through a half-wave plate $(\lambda / 2)$ followed by a polarizing beam splitter $(\mathrm{PBS})$ cube. The linearly polarised beam is collimated and expanded ( $5 \mathrm{~mm}$ in radius) to overfill the back aperture ( $4 \mathrm{~mm}$ in radius) of the microscope objective (Nikon, E Plan $\times 100, \mathrm{NA}=1.25$ in oil, transmission efficiency 0.58 at $1070 \mathrm{~nm}$ ) in order to obtain a diffraction-limited focal spot. A quarter-wave plate $(\lambda / 4)$ placed immediately before the objective creates a circularly polarised light field. Once a single nanovaterite particle is trapped and set into rotation, the transmitted light through the trapped nanoparticle is collected using a condenser lens (Mitutoyo, $\times 50, \mathrm{NA}=0.43$ in air) and directed onto a quadrant photodiode (QPD) array (First Sensor, QP50-6SD2, -3dB at $150 \mathrm{kHz}$ ) after the PBS. Back-focal plane interferometry is used to track the nanoparticle position and the rotation rate (see Detection scheme) at optical powers ranging from $0.05 \mathrm{~W}$ up to $3 \mathrm{~W}$ (measured at the back aperture of the microscope objective, MO).

Experimental protocol. For our experiment, the nanovaterite particles are re-suspended in heavy water (deuterium oxide, $\mathrm{D}_{2} \mathrm{O}$ ). This minimises laser-induced heating of the medium by the absorption of the trapping laser at $532 \mathrm{~nm}$, which is estimated to be $<0.1^{\circ} \mathrm{CW}^{-1}$ 
.${ }^{29,30}$ Syringe filters (PALL, Acrodisc, pore size $1.0 \mu \mathrm{m}$ ) are used to remove particles larger than $1 \mu \mathrm{m}$ in diameter, if necessary, from the sample prior to the trapping experiments. The sample of colloidal suspension is prepared in a miniature cylindrical chamber (circular vinyl sticker spacer of $10 \mathrm{~mm}$ in diameter and $100 \mu \mathrm{m}$ in thickness), which is enclosed with type-1 glass coverslips. Nanoparticles are trapped at an axial distance of $10 \mu \mathrm{m}$ above the glass substrate.

Detection scheme. The rotation rate, $f_{\text {rot }}=\Omega / 2 \pi$ of a trapped and spinning nanovaterite particle is determined from the power spectra obtained from a QPD array. (see Fig. 2) We observe optical beating at $2 f_{\text {rot }}$ when the nanoparticle rotates at $f_{\text {rot }}$ due to the angular Doppler effect. ${ }^{4}$ In practice, $f_{\text {rot }}$ is also detected because of the variation in the photodiode signal induced by a small optical asymmetry of the nanoparticle. The QPD and a photodiode measures the right $\left(P_{R}\right)$ and left $\left(P_{L}\right) \mathrm{CP}$ components of the scattered light, which are used for the determination of the optical torque, $\tau_{\mathrm{opt}}=\Delta \sigma_{\mathrm{CP}} P / \omega$, where $\Delta \sigma_{\mathrm{CP}}=\left(P_{L}-P_{R}\right) / P$, transferred to the particle. ${ }^{2}$

\section{Acknowledgement}

The authors acknowledge funding from the UK Engineering and Physical Sciences Research Council (EPSRC) grants: EP/J01771X/1 and EP/M000869/1, and KD acknowledges support of a Royal Society Leverhulme Trust Senior Fellowship. We thank Professor Takashige Omatsu, Chiba University for useful discussions.

\section{Supporting Information Available}

The following file is available free of charge.

- supp.pdf: supplementary information

This material is available free of charge via the Internet at http://pubs.acs.org/. 


\section{References}

(1) Friese, M. E. J.; Nieminen, T. A.; Heckenberg, N. R.; Rubinsztein-Dunlop, H. Optical Alignment and Spinning of Laser-Trapped Microscopic Particles. Nature 1998, 394, 348-350.

(2) Arita, Y.; McKinley, A. W.; Mazilu, M.; Rubinsztein-Dunlop, H.; Dholakia, K. Picoliter Rheology of Gaseous Media Using a Rotating Optically Trapped Birefringent Microparticle. Anal. Chem. 2011, 83, 8855-8858.

(3) Padgett, M.; Bowman, R. Tweezers with a Twist. Nat. Photonics 2011, 5, 343-348.

(4) Arita, Y.; Mazilu, M.; Dholakia, K. Laser-Induced Rotation and Cooling of a Trapped Microgyroscope in Vacuum. Nat. Commun. 2013, 4, 2374.

(5) Lehmuskero, A.; Ogier, R.; Gschneidtner, T.; Johansson, P.; Käll, M. Ultrafast Spinning of Gold Nanoparticles in Water Using Circularly Polarized Light. Nano. Lett. 2013, 13, 3129-3134.

(6) Brzobohatý, O.; Arzola, A. V.; Šiler, M.; Chvátal, L.; Jákl, P.; Simpson, S.; Zemánek, P. Complex Rotational Dynamics of Multiple Spheroidal Particles in a Circularly Polarized, Dual Beam Trap. Opt. Express 2015, 23, 7273-7287.

(7) Spesyvtseva, S. E. S.; Dholakia, K. Trapping in a Material World. Acs Photonics 2016, 3, 719-736.

(8) Bishop, A. I.; Nieminen, T. A.; Heckenberg, N. R.; Rubinsztein-Dunlop, H. Optical Microrheology Using Rotating Laser-Trapped Particles. Phys. Rev. Lett. 2004, 92, 198104.

(9) Parkin, S. J.; Knöner, G.; Nieminen, T. A.; Heckenberg, N. R.; Rubinsztein-Dunlop, H. Picoliter Viscometry Using Optically Rotated Particles. Phys. Rev. E 2007, 76, 041507.

(10) Seol, Y.; Carpenter, A. E.; Perkins, T. T. Gold Nanoparticles: Enhanced Optical Trapping and Sensitivity Coupled with Significant Heating. Opt. Lett. 2006, 31, 2429-2431. 
(11) Shao, L.; Yang, Z. J.; Andrén, D.; Johansson, P.; Käll, M. Gold Nanorod Rotary Motors Driven by Resonant Light Scattering. ACS Nano 2015, 9, 12542-12551.

(12) La Porta, A.; Wang, M. D. Optical Torque Wrench: Angular Trapping, Rotation, and Torque Detection of Quartz Microparticles. Phys. Rev. Lett. 2004, 92, 190801.

(13) Fernández-Nieves, A.; Cristobal, G.; Garcés-Chávez, V.; Spalding, G. C.; Dholakia, K.; Weitz, D. A. Optically Anisotropic Colloids of Controllable Shape. Adv. Mater. 2005, 17, 680-684.

(14) Vogel, R.; Persson, M.; Feng, C.; Parkin, S. J.; Nieminen, T. A.; Wood, B.; Heckenberg, N. R.; Rubinsztein-Dunlop, H. Synthesis and Surface Modification of Birefringent Vaterite Microspheres. Langmuir 2009, 25, 11672-11679.

(15) Zhao, R. K.; Manjavacas, A.; de Abajo, F. J. G.; Pendry, J. B. Rotational Quantum Friction. Phys. Rev. Lett. 2012, 109, 123604.

(16) Arita, Y.; Mazilu, M.; Vettenburg, T.; Wright, E. M.; Dholakia, K. Rotation of Two Trapped Microparticles in Vacuum: Observation of Optically Mediated Parametric Resonances. Opt. Lett. 2015, 40, 4751-4754.

(17) Norris, D. A.; Puri, N.; Sinko, P. J. The Effect of Physical Barriers and Properties on the Oral Absorption of Particulates. Adv. Drug Deliver. Rev. 1998, 34, 135-154.

(18) Rings, D.; Chakraborty, D.; Kroy, K. Rotational Hot Brownian Motion. New J. Phys. 2012, 14, 053012 .

(19) Parkin, S. J.; Vogel, R.; Persson, M.; Funk, M.; Loke, V. L. Y.; Nieminen, T. A.; Heckenberg, N. R.; Rubinsztein-Dunlop, H. Highly Birefringent Vaterite Microspheres: Production, Characterization and Applications for Optical Micromanipulation. Opt. Express 2009, 17, 21944-21955. 
(20) Bogdan V. Parakhonskiy, A. H.; Antolini, R. Sub-Micrometer Vaterite Containers: Synthesis, Substance Loading, and Release. Angew. Chem. Int. Ed. 2012, 51, 11951197.

(21) Kestin, J.; Sokolov, M.; Wakeham, W. A. Viscosity of Liquid Water in Range $-8^{\circ} \mathrm{C}$ to $150^{\circ}$ C. J. Phys. Chem. Ref. Data 1978, 7, 941-948.

(22) Incropera, F. P.; DeWitt, D. P. Fundamentals of Heat and Mass Transfer; Wiley: New York, 1996.

(23) Panton, R. L. Incompressible Flow, 3rd ed.; Wiley: New York; Chichester, 2013.

(24) Arita, Y.; Richards, J. M.; Mazilu, M.; Spalding, G. C.; Spesyvtseva, S. E. S.; Craig, D.; Dholakia, K. Data underpinning - Rotational Dynamics and Heating of Trapped Nanovaterite Particles 2016, http://dx.doi.org/10.17630/842a9448-09b2-4f66-bd527cd020fdef2d.

(25) Le Neindre, B.; Bury, P.; Tufeu, R.; Vodar, B. Thermal Conductivity Coefficients of Water and Heavy Water in the Liquid State up to $370^{\circ}$ C. J. Chem. Eng. Data 1976, 21, 265-274.

(26) Kelf, T. A.; Sreenivasan, V. K. A.; Sun, J.; Kim, E. J.; Goldys, E. M.; Zvyagin, A. V. Non-Specific Cellular Uptake of Surface-Functionalized Quantum Dots. Nanotechnology 2010, 21, 285105.

(27) Wu, T.; Nieminen, T. A.; Mohanty, S.; Miotke, J.; Meyer, R. L.; RubinszteinDunlop, H.; Berns, M. W. A Photon-Driven Micromotor Can Direct Nerve Fibre Growth. Nat. Photonics 2012, 6, 62-67.

(28) Roder, P. B.; Smith, B. E.; Zhou, X.; Crane, M. J.; Pauzauskie, P. J. Laser Refrigeration of Hydrothermal Nanocrystals in Physiological Media. Proc. Natl. Acad. Sci. U.S.A. 2015, 112, 15024-9. 
(29) Peterman, E. J. G.; Gittes, F.; Schmidt, C. F. Laser-Induced Heating in Optical Traps. Biophys. J. 2003, 84, 1308-1316.

(30) Kedenburg, S.; Vieweg, M.; Gissibl, T.; Giessen, H. Linear Refractive Index and Absorption Measurements of Nonlinear Optical Liquids in the Visible and Near-Infrared Spectral Region. Opt. Mater. Express 2012, 2, 1588-1611. 
Figure 1: SEM images of nanovaterite crystals. (a) A selection of nanoparticles (b) An expanded view of the enclosed field with dashed line boundary.

Figure 2: Power spectral density (PSD) of a rotating nanovaterite particle showing a rotation rate, $f_{\text {rot }}$ of $4.9 \mathrm{kHz}$ and its high harmonics of $2 f_{\text {rot }}$ and $3 f_{\text {rot }}$.

Figure 3: Nanoparticle rotation rate, $f_{\text {rot }}$ and trapping corner frequency $f_{\mathrm{c}}$ as a function of the incident optical power. (a) Measured data ( $f_{\text {rot }}$ represented by blue open circles and $f_{\mathrm{c}}$ by green open squares) together with model results ${ }^{24}$ (curved solid lines take heating into account and dashed lines neglect heating). (b) Nanoparticle surface temperature, $T_{s}$ as a function of the dissipated power using a finite element approach ${ }^{24}$ and an analytical approximation (Eq. 1).

Figure 4: Temperature dependent dynamic viscosity correction factor. ${ }^{24}$ (a) Radial temperature profile and the corresponding dynamic viscosity of heavy water surrounding a nanoparticle dissipating $100 \mu \mathrm{W}$. (b) Viscosity correction factors (as defined in the main text) for rotational (blue solid line) and translational (green solid line) motion of the nanoparticle (radius of $423 \mathrm{~nm}$ ) as a function of its surface temperature. 


\section{Graphical TOC Entry}

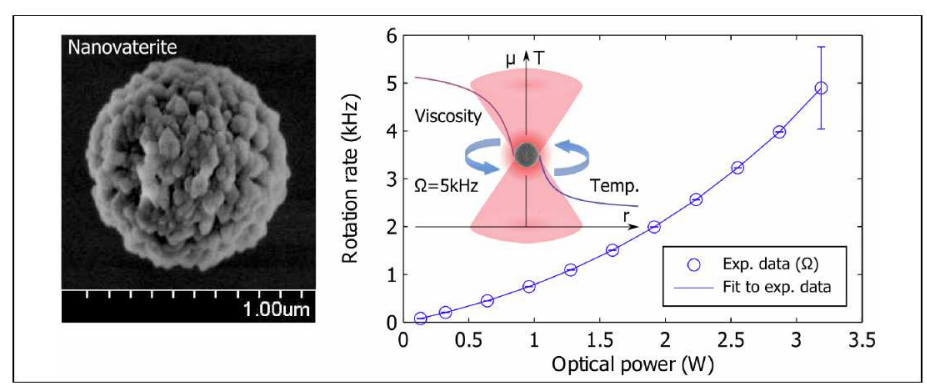

15
16

17 